\title{
Infant antibiotic use and wheeze and asthma risk: a systematic review and meta-analysis
}

\author{
J. Penders*\#, I. Kummeling ${ }^{\#, \text { a }}$ and C. Thijs
}

ABSTRACT: Our aim was to systematically review and meta-analyse longitudinal studies on antibiotic use and subsequent development of wheeze and/or asthma with regards to study quality, outcome measurement, reverse causation (RC; wheezing/asthma symptoms have caused prescription of antibiotics) and confounding by indication (Cbl; respiratory tract infections leading to antibiotic use may be the underlying cause triggering asthma symptom development).

English-language papers and studies published before November 1, 2010 with longitudinal observational design were included. Study quality was assessed using the Newcastle-Ottawa scale.

We identified 21 longitudinal studies. The effect of antibiotic use on wheeze/asthma risk varied between studies. 18 studies were eligible for meta-analysis showing pooled OR 1.27 (95\% Cl 1.121.43) for wheeze/asthma. When we eliminated studies with possible $R C$ and $C b l$, the pooled risk estimate in the nine remaining studies was attenuated to OR 1.12 (95\% $\mathrm{Cl} 0.98-1.26)$. Definition of wheeze/asthma and age at follow-up differed between studies. Three studies focused on wheeze/ asthma beyond 5-6 yrs of age with the presence of active symptoms and/or medication (pooled OR 1.08, 95\% Cl 0.93-1.23; dominated by one study).

$\mathrm{RC}$ and $\mathrm{Cbl}$ lead to overestimation of the association between antibiotic use and subsequent development of wheeze/asthma. Association was weak when fully adjusted for these types of bias. Heterogeneity of disease definition between studies could affect the results.

KEYWORDS: Allergy, antibiotics, asthma, breastfeeding, infant, meta-analysis

$\mathbf{R}$ eports on the relationship between antibiotic use in infancy and the subsequent development of wheeze or asthma have been published continuously for over a decade.

Conflicting results may be attributable to different study designs (cross-sectional or longitudinal), differences in outcome definition and population age at follow-up, and two forms of bias related to causality (reverse causation (RC) and confounding by indication $(\mathrm{CbI}))$ [1].

Cross-sectional studies may be prone to bias because they do not allow an assessment of the timing of exposure in relation to the occurrence of wheeze or asthma and, therefore, longitudinal (cohort) studies are preferred. Indeed, a recent meta-analysis showed a significantly stronger association between infant antibiotic use and asthma in retrospective and cross-sectional studies, as compared to prospective studies [2].
Caution is still needed in cohort studies before concluding that associations are causal. This study addresses two issues of causality: RC and CbI [1]. RC plays a role when the period of antibiotic use and the period that wheezing/ asthmatic symptoms appeared (partly) coincide. In that situation the symptoms themselves may have caused the prescription of antibiotics.

$\mathrm{CbI}$ refers to those situations in which the indication for treatment acts as a confounder. In those cases, an association between antibiotic intake and wheeze/asthma is confounded (explained) by a third factor that is an indication for antibiotic prescription while at the same time being a risk factor for asthma. This may occur when antibiotics are prescribed for respiratory tract infections which then are the actual risk factor for wheeze/asthma.

Some recent studies [3] and narrative reviews [1] even focused on the very question of whether RC

\section{AFFILIATIONS}

*Dept of Medical Microbiology, NUTRIM School for Nutrition, Toxicology and Metabolism, Maastricht University Medical Centre \#Dept of Epidemiology, CAPHRI School of Public Health and Primary Care, Maastricht University, Maastricht, The Netherlands. 'Respiratory Epidemiology and Public Health, National Heart and Lung Institute, Imperial College London, London, UK.

\section{CORRESPONDENCE}

J. Penders

Dept of Medical Microbiology

Maastricht University Medical Centre P.0 Box 5800

NL-6202 AZ

Maastricht

The Netherlands

E-mail: j.penders@

maastrichtuniversity.n!

Received:

July 062010

Accepted after revision: Jan 022011

First published online:

Jan 132011 
or $\mathrm{CbI}$ could explain the reported associations between antibiotic use and respiratory allergy and asthma, without deriving a conclusive answer.

Heterogeneity in outcome definition might contribute to inconsistency in results. Asthma is a heterogeneous condition and wheezing, its major clinical expression, is a nonspecific symptom associated with airflow obstruction through narrowed airways.

Infant wheezing is often transient and resolves between 3 and 5 yrs of age. Furthermore, early life wheezing is frequently associated with viral respiratory agents, often respiratory syncytial virus [4]. Therefore, studies focusing on pre-school wheeze represent different and more heterogeneous patient populations compared to studies that focus on wheeze-related or asthma-like symptoms that persist into school age.

In this study we systematically reviewed the available longitudinal studies and describe how outcome definition, RC and $\mathrm{CbI}$ affected the association between antibiotic use in early life and the subsequent development of wheeze or asthma.

\section{METHODS}

\section{Systematic review}

We included longitudinal observational studies on the association between antibiotic exposure and wheeze/asthma risk: historical or prospective cohort studies and nested casecontrol studies within cohort or case-cohort studies. Crosssectional and retrospective case-control studies were excluded.

Medline, EMBASE and PubMed were searched up to November 1, 2010 using the following keywords: (antibiotic* or antibact*) AND (allergy OR allergic OR hypersens* OR atop* OR eczema OR asthma OR wheeze) AND (cohort OR follow-up OR longitudinal OR prospective). The search was limited to English-language papers. Additional studies were found by searching reference lists of relevant articles.

\section{Meta-analyses}

To assess how outcome definition, $\mathrm{RC}$ and $\mathrm{CbI}$ affect the association between antibiotic use in early life and the subsequent development of wheeze or asthma, we conducted three meta-analyses.

\section{Inclusion criteria}

The first "overall" meta-analysis included all studies from the systematic review that fulfilled the following additional criteria. 1) Assessment of antibiotic exposure: information on antibiotic exposure collected prospectively; exposure in first year of life through direct oral administration to the infant; time lag between antibiotic exposure and assessment of this exposure (e.g. parental report) $\leqslant 1$ yr (to avoid recall bias) or historically recorded exposure data were available (e.g. from prescription registry). 2) Assessment of outcome(s): wheezing/ asthmatic symptoms in first 10 yrs of life (studies in children $>10$ yrs of age, adolescents and adults were excluded); wheeze and/or asthma included as a primary outcome measure. 3) Measures of association and adjustment for confounding: measures of association (odds ratios (OR), relative risk (RR) or hazard ratio (HR)) were available along with parameters of precision needed for statistical pooling (e.g. 95\% confidence interval and standard error); adjustment for potential confounding factors by stratified or multivariable analysis.

\section{Data extraction}

Subsequently, exposure and outcome measures were selected as follows. 1) Antibiotic exposure: comparisons for any versus no antibiotic use were selected. If unavailable but associations were presented according to the number of antibiotic prescriptions, the association comparing the highest number of antibiotic courses versus no antibiotic courses was selected. 2) Wheeze and/or asthma outcomes: when studies presented respiratory outcomes at different ages, the oldest age/longest duration of follow-up was selected. When multiple time-points of wheezing/asthmatic symptoms were presented ("ever" versus "current"), "current" symptoms were selected to avoid $\mathrm{RC}$. When associations for multiple respiratory outcomes were presented, the first applicable option from the following list was selected: doctor's diagnosed asthma; reported asthma; reported wheeze with asthma medication; reported persistent wheeze; reported wheeze; reported transient wheeze.

\section{Additional meta-analyses}

We then conducted a second meta-analysis restricting the studies of the "overall" meta-analyses to studies that render bias from RC and CbI unlikely. 1) Avoidance of RC: no overlap between the exposure and development of first wheezing/ asthmatic symptoms in statistical analysis. 2) Avoidance of $\mathrm{CbI}$ : adjusting the analysis for lower respiratory tract infections (LRTIs) (or "chest infections").

If this was not entirely clear from the publications, we contacted the authors via e-mail for clarification. All authors promptly responded to our queries with satisfactory answers.

Finally, we conducted a third meta-analysis restricting the studies from the "overall" meta-analyses to studies that focused on wheeze/asthma beyond the age of 5-6 yrs and that included the presence of active symptoms and/or medication use at school age.

Two of our group (J. Penders and I. Kummeling) independently assessed which studies were eligible for the overall and "strict criteria" meta-analyses, and in case of a conflicting judgment a third author (C. Thijs) was consulted. In all cases consensus was reached.

\section{Quality assessment of studies in meta-analysis}

Articles eligible for inclusion in the overall meta-analysis were independently assessed for quality by three reviewers (J. Penders, I. Kummeling and C. Thijs) using the NewcastleOttawa Quality Assessment Scale (NOS) for cohort studies [5].

The reviewers resolved disagreements by discussion to achieve consensus. For a detailed description of the quality assessment according to the NOS refer to the supplementary material.

\section{Statistical analyses}

Measures of association (OR, RR and HR) and their 95\% confidence intervals were abstracted or derived by using data reported in the publications. When several measures of association were reported, we selected the measure according to the criteria mentioned above. When RRs or HRs were 
presented, we assumed that the OR approximated the RR or HR and treated them as such.

To derive a pooled OR from individual studies we used a random-effects meta-analysis model. Heterogeneity was quantified with the $\mathrm{I}^{2}$ index, which describes the proportion of total variation in study estimates due to heterogeneity [6]. To assess the potential presence of publication bias, the size of the estimated effect of the included studies was plotted against the standard error (funnel plot). Begg's rank correlation [7] and Egger's linear regression tests [8] were used to detect asymmetry in funnel plots. Statistical analyses were conducted with Stata version 10.1 (STATA Corp., College Station, TX, USA).

\section{RESULTS}

\section{Study selection and characteristics}

We identified 634 papers, of which 35 were original studies on the association between antibiotic exposure and the risk of allergic diseases and/or wheeze and/or asthma (fig. 1).

15 studies were excluded for the following reasons: antibiotic exposure was collected retrospectively $(n=4)$ [9-12]; no report on wheeze and/or asthma, but other allergic outcomes only $(n=6)$ [13-18]; no association measure for wheeze or asthma separately but an overall outcome called "atopy" which also included eczema and/or hay fever $(n=1)$ [19]; only prenatal antibiotic use reported $(n=3)$ [20-22]; and adult population $(n=1)$ [23].

Searching reference lists resulted in the identification of eight additional studies, of which one was an ecological study [24] and six were cross-sectional studies [25-30]; one met our inclusion criteria [31]. Altogether this resulted in 21 studies fulfilling our selection criteria (table E1 in supplementary material).

\section{Systematic review}

In seven of these 21 studies wheeze or asthma were not associated with antibiotic use [31-37], so that bias deriving from $\mathrm{RC}$ and $\mathrm{CbI}$ was not of concern. We will go on to discuss the remaining 14 studies with regard to $\mathrm{RC}$ and $\mathrm{CbI}$.

\section{Reverse causation}

In two studies, exposure and outcome partly coincided and RC could not be excluded [38, 39]. Four other studies [3, 40-42] corrected for RC by means of excluding children who had already developed symptoms at the time of antibiotic use from the analyses. In three of these studies [3, 40, 42], initially positive associations between antibiotic use and wheeze or asthma disappeared after correction, whereas in the fourth study [41] the association between antibiotic use and wheeze remained after correcting for RC. RC as an explanation was unlikely in six studies that had ensured that exposure (antibiotic use) and follow-up periods took place subsequently and were not overlapping [43-48]. Furthermore, ALM et al. [49] found an increased risk of wheeze in the first year of life after antibiotic use in the first days after birth. This is such a short period of overlap that we also deemed RC here unlikely.

Taken together, this results in two studies with doubtful results $[38,39]$, three studies in which the association disappeared after correcting for RC [3, 40,42], and eight that found a positive association that could not be explained by RC [41, 43-49].

Finally, the study by MAI et al. [50] eliminated CbI before tackling the influence of RC. Therefore, this study will only be discussed in the following section, together with the remaining eight studies in which the positive association could not be explained by RC.

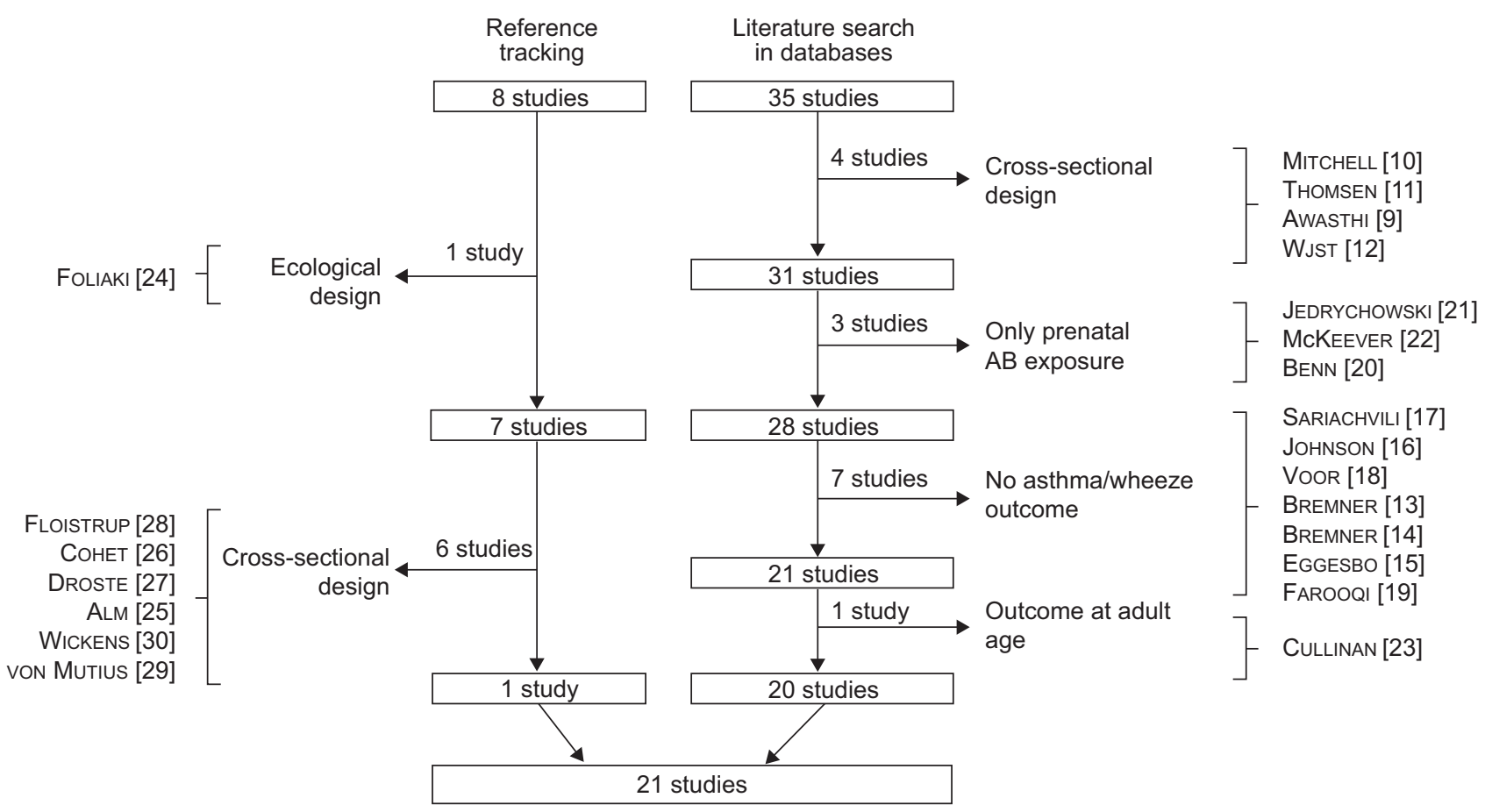

FIGURE 1. Results of the literature search and selection of studies. AB: antibiotic. 


\section{Confounding by indication}

Several of the eight studies mentioned previously (RC excluded) did not collect relevant information about the indication for antibiotic prescription or episodes of infections at the time of antibiotic exposure [41, 43, 48, 49], whereas the other studies did [44-47]. The study by KUSEL et al. [46] illustrates why $\mathrm{CbI}$ should be taken into account. In this study children with LTRI and wheeze had used antibiotics twice as often as children with LTRI without wheeze or a higher tract respiratory infection (HTRI). The children with LTRI and wheeze had a three-fold higher risk of developing asthma up to $5 \mathrm{yrs}$ of age. After adjustments for the indication for antibiotic use (LTRI with wheeze, without wheeze, HTRI, or other symptoms such as fever) the association between antibiotic use and asthma diagnosis almost entirely disappeared. This clearly illustrates $\mathrm{CbI}$ and indicates that it may not be a direct causal effect of antibiotics. It is more likely that patients with an underlying susceptibility to react to infections with wheeze are more prone to receive antibiotics.

In the study by MAI et al. [50], associations between antibiotic use in the first year of life and wheeze/asthma at 4-8 yrs of age became weaker after adjustment for respiratory infections, and only remained significant for the outcomes at 4 yrs of age. Following exclusion of children with wheeze, asthma and eczema symptoms in their first year of life, thus eliminating $\mathrm{RC}$, the associations between antibiotic use and wheeze and asthma at 4 and 8 yrs of age were completely eliminated [50].

Two studies adjusted for consultation behaviour or number of illness visits $[43,48]$, and one of them expressly stated that this was to resolve $\mathrm{CbI}$ [43]; however, we contend that this method is not specific enough for the exact indication for antibiotics.

Finally, in three other studies, the associations between antibiotic use and asthma remained after adequate adjustment for infections [44, 45, 47].

Taken together, this results in four studies that did not collect data on the indication for antibiotic prescription or episodes of LRTIs [41, 43, 48, 49], two studies in which the association disappeared after adjusting for $\mathrm{CbI}[46,50]$ and three studies in which a positive association remained after controlling for both $\mathrm{RC}$ and $\mathrm{CbI}[44,45,47]$.

\section{Outcome definition}

Studies differed widely regarding the outcome definition and age at follow-up. Only three studies examined the development of (recurrent) wheeze in the first or 2 yrs of life [40, 41, 49]. It is worth noting that these are the studies which reported by far the strongest associations with antibiotic use (fig. 2a).

Six studies had follow-up periods until $4-5$ yrs of age $[3,32,34$, $35,43,46$,] of which one focused on recurrent wheeze [32] and the remaining five were based on the outcome "asthma" using various definitions $[3,34,35,43,46]$. It has to be kept in mind that a diagnosis of asthma in pre-school children is difficult to establish, as it often cannot be distinguished from infectionrelated wheeze. As a consequence this may result in serious misclassification of transient wheezers as asthma cases. One study focused on transient wheeze, excluding those children with persistent wheezing up to 6 yrs of age [38].
Although several studies had a follow-up beyond pre-school age $[36,42,44,45,47,48,50]$, several of them did not distinguish between age periods of diagnosis, thus still resulting in many cases already being diagnosed at pre-school ages [36, 44, 45, 48]. The studies by MARTEL et al. [45] and PONSONBY et al. [36] did conduct subgroup analysis based on age of asthma onset, but with a cut-off of 3 and 4 yrs, respectively. The studies by MCKeEver et al. [48] and MARRA et al. [44], with a follow-up of 9-11 yrs, used survival analysis but the vast majority of asthma cases were diagnosed before the age of 5 yrs and, therefore, did not agree to our criterion for the third meta-analysis.

This leaves only three studies that explicitly focused on the persistence of wheeze or asthma into school age. These studies, with follow-up until the age of 7-8 yrs, all included current (past 12 months) wheezing/asthmatic symptoms and/or current medication use in their outcome definition $[42,47,50]$.

\section{Meta-analysis}

18 studies on wheeze and asthma were included in the overall meta-analysis. Table 1 shows the quality grading according to the NOS. The pooled risk estimate was OR 1.27, 95\% CI 1.121.43 (fig. 2a), indicating a slight increase in the risk of developing asthma and/or wheeze after antibiotic use in the first year of life. There is no indication of publication bias as both the Begg's test $(p=0.85)$ and the Egger's test $(p=0.14)$ do not indicate asymmetry of the funnel plot (fig. 3). It has to be noted that publication bias cannot be ruled out completely because of the low sensitivity of both tests for $<20$ studies [51]. The results were greatly variable between studies, and the test for heterogeneity was statistically significant $\left(\mathrm{I}^{2}\right.$ index $75.7 \%$; test for heterogeneity $\mathrm{p}<0.001)$.

Nine studies fulfilled the criteria for inclusion in the first restricted meta-analysis ( $\mathrm{RC}$ and $\mathrm{CbI}$ unlikely) (fig. 2b).With the exception of the study by Dom et al. [32], these studies were also the studies that scored highest on the NOS. The pooled OR was 1.12 (95\% CI 0.98-1.26) and the results showed less heterogeneity ( $\mathrm{I}^{2}$ decreased to $46.3 \%$; test for heterogeneity $p=0.06$ ). This pooled OR was substantially lower than the OR in the overall meta-analysis, but because the OR was still somewhat greater than 1.0, it indicates that potential bias from $\mathrm{RC}$ and $\mathrm{CbI}$ does not entirely explain the increased risk. However, the lower 95\% confidence interval of 0.98 indicates that an OR of 1.0 (no effect) cannot be excluded with certainty.

Only three studies fulfilled the inclusion criterion, persistence of wheeze/asthma into school age, for the third meta-analysis. Since the study by HARRIS et al. [42] contributed $>80 \%$ to the weight, the pooled OR $(1.08,95 \%$ CI $0.93-1.23)$ was dominated and closely resembled the association from this study (OR 1.08, 95\% CI 0.94-1.25) (fig. E1 in supplementary material).

\section{DISCUSSION}

We aimed to investigate how two methodological concepts, namely $\mathrm{RC}$ and $\mathrm{CbI}$, affect the potential association between antibiotic use in early life and the subsequent development of asthma/wheezing. Thus, we compared the pooled overall effect size of a "strict criteria" meta-analysis (RC and CbI unlikely) with that of the overall meta-analysis that included welldesigned studies but in which $\mathrm{RC}$ and $\mathrm{CbI}$ could not be 
a)

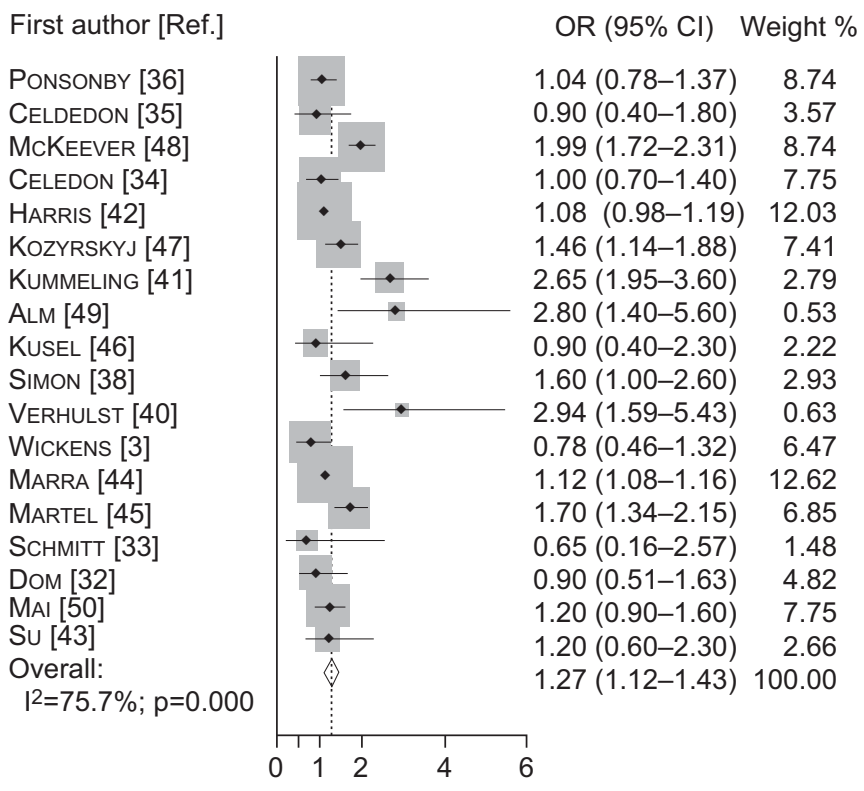

b)

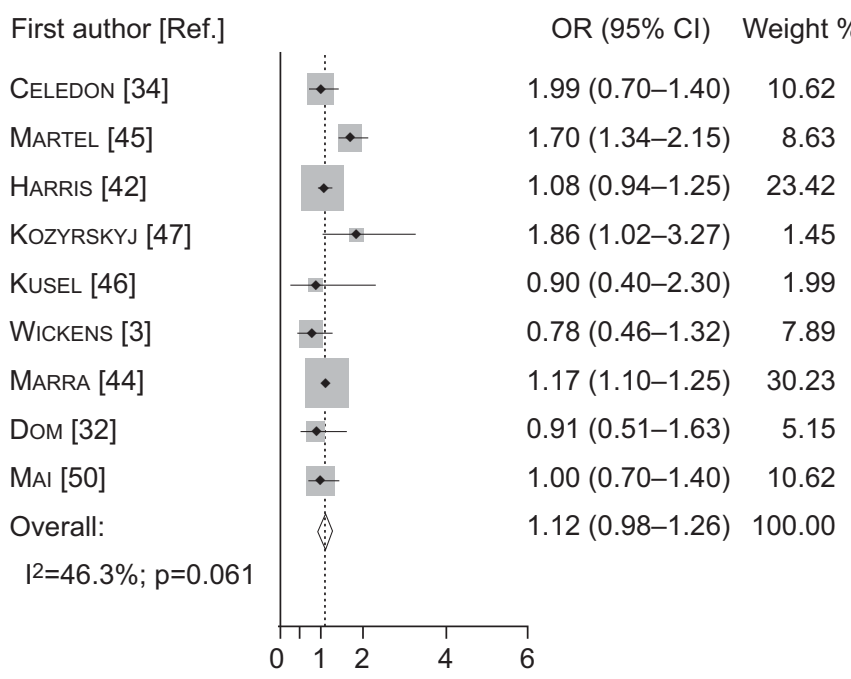

FIGURE 2. a) Overall and b) strict criteria meta-analysis of studies of antibiotic use in childhood and risk of asthma and/or wheeze. Note that the weights are from random effects analysis.

excluded. Furthermore, we studied the effect of heterogeneity in the disease definition on the outcome in a third meta-analysis.

In the overall meta-analysis the overall pooled estimate indicated a small but statistically significantly increased risk (pooled OR 1.27). When this was restricted to the nine studies that met the strict criteria for exclusion of $\mathrm{RC}$ and $\mathrm{CbI}$, the pooled estimate fell to 1.12, which lost statistical significance.

Heterogeneity in the definition of asthma may affect the results. A reliable diagnosis of asthma can only be established beyond 5-6 yrs of age. Four studies focused specifically on wheeze in early childhood [38, 40, 41, 49] and none of them were included in the strict meta-analysis. Three studies focused on active symptoms/medication use at school age
[50, 47, 42] and all three were included in the "strict criteria" meta-analysis. The other studies did not follow-up until school age $[3,32,34,46]$ or did follow-up until school age but did not (profoundly) distinguish between those cases with only symptoms at pre-school age and those who persisted to have symptoms into school age [2, 45]. Thus, in the strict metaanalysis the definition of asthma is heterogeneous and one should be cautious drawing any firm conclusion on the association between antibiotic use and true asthma.

To further narrow down to true asthma, we conducted a third meta-analysis that only included studies focusing on active wheeze/asthma at school age. Unfortunately, only three studies could be included in this meta-analysis, resulting in an unstable effect size largely influenced by only one study.

Therefore, more studies are needed with a follow-up long enough to reliably define asthma and with a minimal risk for bias due to $\mathrm{RC}$ and $\mathrm{CbI}$.

The negative impact of antibiotics on the gastrointestinal microbiota is often referred to as the underlying biological mechanism explaining the potential causal effect of antibiotic use on asthma. Administration of antimicrobial agents causes disturbances in the ecological balance between the host and the normal intestinal microbiota and also leads to selective overgrowth of opportunistic pathogens such as Clostridium difficile or Candida albicans [52]. The "microflora hypothesis" states that the association between antibiotic use and asthma is caused by the negative impact on the gastrointestinal microbiota [53].

As the infant intestinal microbiota plays a crucial role in the development of immune regulation and oral tolerance induction [54], disruption of the microbiota might also disrupt oral tolerance, potentially by interfering with the dendritic cells that promote antigen-specific regulatory T-cell responses [55]. Epidemiological studies have indeed shown differences in gut microbiota composition of allergic and non-allergic children [56].

If the microbiota is the causal link in the association between antibiotic use and asthma/wheezing, then timing, dosage and type of antibiotics would all influence this association especially in early life; when the immune system is still immature, the gut microbiota is thought to play an important role in immuneregulation [54]. Factors influencing the microbiota in early life may have a profound effect on the mature microbial composition [57] and gut microbiota (and possibly also the airway microbiota) is more heavily disturbed by broad-spectrum than small-spectrum antibiotics.

Regarding the timing of exposure to antibiotics we restricted our analysis to antibiotic prescriptions in the first year of life. Some studies investigated very early age, with one study reporting an increased risk of wheezing in children exposed to antibiotics in the neonatal period [49], while the other studies determining antibiotic use in the first weeks or months did not find an association $[3,36,37,40]$.

Several studies included the number of courses of antibiotic in their analysis [34, 35, 43, 44, 46-50]. In three studies, the highest risk for asthma/wheezing was found in children exposed to more than four courses of antibiotics in the first year of life 
TABLE 1 Assessment of methodological quality of studies according to the Newcastle-Ottowa Scale for cohort studies

\begin{tabular}{|c|c|c|c|c|c|}
\hline First author [Ref.] & Country & Selection ${ }^{\#}$ & Comparability & Outcome $^{+}$ & $\begin{array}{c}\text { Included in strict criteria } \\
\text { analysis }\end{array}$ \\
\hline KOZYRSKYJ [47] & Canada & $* * * *$ & $* *$ & $* * *$ & Yes \\
\hline MARTEL [45] & Canada & $* * * *$ & $* *$ & $* *$ & Yes \\
\hline HARRIS [42] & UK & $* * * *$ & $* *$ & $* *$ & Yes \\
\hline WICKENS [3] & New Zealand & $* * * *$ & $* *$ & * & Yes \\
\hline Kusel [46] & Australia & $* * * *$ & $* *$ & * & Yes \\
\hline PonsonBy [36] & USA & $* * * *$ & $*$ & $* *$ & No \\
\hline Su [43] & USA & $* * * *$ & $*$ & $*$ & No \\
\hline Sснмітт [33] & Germany & $* * *$ & $*$ & $* *$ & No \\
\hline McKeever [48] & UK & $* * * *$ & * & $*$ & No \\
\hline Dом [32] & Belgium & $* *$ & $* *$ & & Yes \\
\hline SIMON [38] & USA & $* *$ & * & & No \\
\hline Verhulst [40] & Belgium & $* *$ & $*$ & & No \\
\hline
\end{tabular}

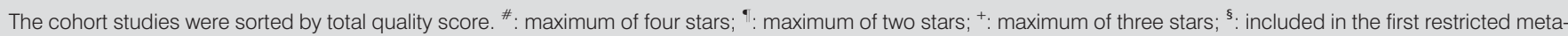
analysis (reverse causation and confounding by indication unlikely).

[44, 47, 48], while in the remaining studies no (dose-response) association was found [34, 35, 43, 46, 49, 50].

Information on the classes and spectrum of antibiotics was available in five studies [33, 39, 44, 47, 48]. One study found a positive association between broad-spectrum antibiotic use and atopic wheeze [39] and in another study the broader spectrum antibiotics produced a stronger increased risk for asthma than the narrow-spectrum antibiotics [48]. The influence of type of antibiotics was difficult to interpret in the remaining three studies for various reasons such as very small numbers, circumstantial evidence or very small effect sizes [33, 34, 44].

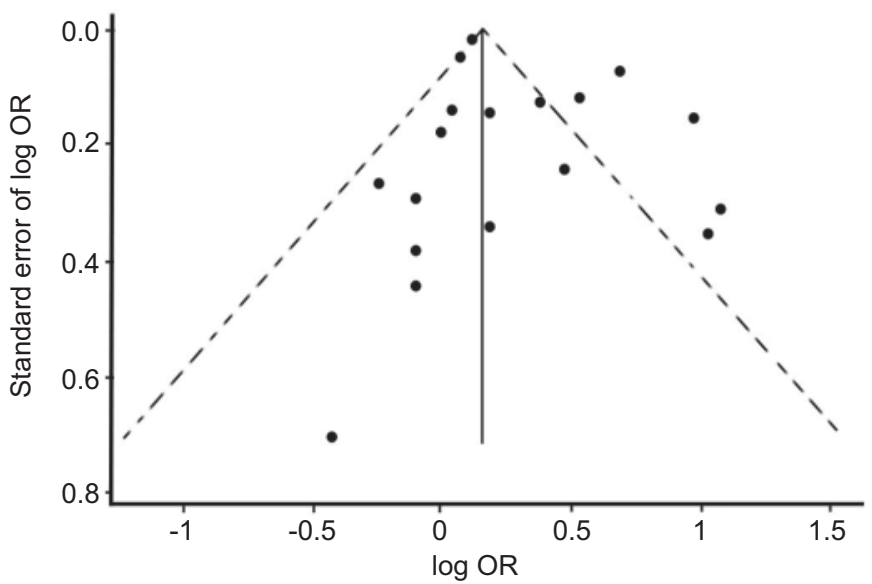

FIGURE 3. Begg's funnel plot with pseudo $95 \%$ confidence limits of all 18 studies included in the overall meta-analysis.
Of note, the lack of association between antibiotic use and allergic sensitisation [3, 23, 31, 35, 41, 42, 46], as reported in the majority of the studies, is not in favour of a causal effect of antibiotics on asthma development.

In conclusion, wheeze and asthma were related to antibiotic use in approximately half of the identified longitudinal studies. $\mathrm{RC}$ and $\mathrm{CbI}$ by respiratory tract infections could explain these associations in many, but not all, of these studies.

A causal effect cannot be dismissed completely, and the disturbing influence of antibiotics on gut or airway microbiota may explain the effect. Alternatively, the observed association in the "strict" meta-analysis could still be due to confounding by consultation behaviour or residual confounding by other factors. The opportunities that currently exist in clinical trials should be used well by inclusion of asthma as adverse outcomes, for instance in trials after the long-term effects of prophylactic antibiotic use, such as after premature rupture of amniotic membranes (ORACLE study) [58]. Other opportunities exist in studies on the prophylactic effects of probiotics on antibiotic-associated diarrhoea in children. These have shown to be promising but have not yet proved effective [59] and have not yet investigated the long-term effects on asthma.

We recommend that new prospective observational studies on the association between antibiotic exposure in early life and subsequent wheeze and asthma should be designed in such a way that bias due to $\mathrm{RC}, \mathrm{CbI}$ and confounding by consultation behaviour is minimised. Collecting detailed information on the number of antibiotic courses, the indication for prescription of the antibiotics and the type of antibiotics prescribed is crucial. It may also be worthwhile to narrow the exposure to the first 6 months 
of life, as the window of opportunity for modulating the infant's immune system may not span the entire first year of life.

Regarding the outcome assessment, follow-up until the age of at least 5-6 yrs is recommended; as only then can a distinction be made between children who only suffered from transient wheeze and those who went on to develop persistent wheeze and asthma. We especially recommend extending the followup of existing well-designed cohort studies reviewed herein.

\section{SUPPORT STATEMENT}

J. Penders is supported by a grant from the Netherlands Asthma Foundation (3.2.07.022). I. Kummeling is supported by a grant from the Thrasher Research Fund.

\section{STATEMENT OF INTEREST}

None declared.

\section{REFERENCES}

1 Kummeling I, Thijs C. Reverse causation and confounding-byindication: do they or do they not explain the association between childhood antibiotic treatment and subsequent development of respiratory illness? Clin Exp Allergy 2008; 38: 1249-1251.

2 Marra F, Lynd L, Coombes M, et al. Does antibiotic exposure during infancy lead to development of asthma? A systematic review and meta-analysis. Chest 2006; 129: 610-618.

3 Wickens K, Ingham T, Epton M, et al. The association of early life exposure to antibiotics and the development of asthma, eczema and atopy in a birth cohort: confounding or causality? Clin Exp Allergy 2008; 38: 1318-1324.

4 Stein RT, Martinez FD. Asthma phenotypes in childhood: lessons from an epidemiological approach. Paediatr Respir Rev 2004; 5: 155-161.

5 Wells GA, Shea B, O'Connell D, et al. The Newcastle-Ottawa Scale (NOS) for assessing the quality of nonrandomised studies in metaanalyses. Available from: www.ohri.ca/programs/clinical_epide miology/oxford.asp Date last accessed: October 29, 2010.

6 Huedo-Medina TB, Sanchez-Meca J, Marin-Martinez F, et al. Assessing heterogeneity in meta-analysis: Q statistic or $\mathrm{I}^{2}$ index? Psychol Methods 2006; 11: 193-206.

7 Begg CB, Mazumdar M. Operating characteristics of a rank correlation test for publication bias. Biometrics 1994; 50: 1088-1101.

8 Egger M, Davey Smith G, Schneider M, et al. Bias in meta-analysis detected by a simple, graphical test. BMJ 1997; 315: 629-634.

9 Awasthi S, Kalra E, Roy S, et al. Prevalence and risk factors of asthma and wheeze in school-going children in Lucknow, North India. Indian Pediatr 2004; 41: 1205-1210.

10 Mitchell EA, Robinson E, Black PN, et al. Risk factors for asthma at 3.5 and 7 years of age. Clin Exp Allergy 2007; 37: 1747-1755.

11 Thomsen SF, Ulrik CS, Porsbjerg C, et al. Early life exposures and risk of atopy among Danish children. Allergy Asthma Proc 2006; 27: 110-114.

12 Wjst M, Hoelscher B, Frye C, et al. Early antibiotic treatment and later asthma. Eur J Med Res 2001; 6: 263-271.

13 Bremner SA, Carey IM, DeWilde S, et al. Early-life exposure to antibacterials and the subsequent development of hayfever in childhood in the UK: case-control studies using the General Practice Research Database and the Doctors' Independent Network. Clin Exp Allergy 2003; 33: 1518-1525.

14 Bremner SA, Carey IM, DeWilde S, et al. Vaccinations, infections and antibacterials in the first grass pollen season of life and risk of later hayfever. Clin Exp Allergy 2007; 37: 512-517.

15 Eggesbo M, Botten G, Stigum H, et al. Is delivery by Cesarean section a risk factor for food allergy? J Allergy Clin Immunol 2003; 112: 420-426.
16 Johnson CC, Ownby DR, Alford SH, et al. Antibiotic exposure in early infancy and risk for childhood atopy. J Allergy Clin Immunol 2005; 115: 1218-1224.

17 Sariachvili M, Droste J, Dom S, et al. Is breast feeding a risk factor for eczema during the first year of life? Pediatr Allergy Immunol 2007; 18: 410-417.

18 Voor T, Julge $\mathrm{K}$, Bottcher MF, et al. Atopic sensitization and atopic dermatitis in Estonian and Swedish infants. Clin Exp Allergy 2005; 352: 153-159.

19 Farooqi IS, Hopkin JM. Early childhood infection and atopic disorder. Thorax 1998; 53: 927-932.

20 Benn CS, Thorsen P, Jensen JS, et al. Maternal vaginal microflora during pregnancy and the risk of asthma hospitalization and use of antiasthma medication in early childhood. I Allergy Clin Immunol 2002; 110: 72-77.

21 Jedrychowski W, Galas A, Whyatt R, et al. The prenatal use of antibiotics and the development of allergic disease in one year old infants. A preliminary study. Int J Occup Med Environ Health 2006; 19: 70-76

22 McKeever TM, Lewis SA, Smith C, et al. The importance of prenatal exposures on the development of allergic disease: a birth cohort study using the West Midlands General Practice Database. Am J Respir Crit Care Med 2002; 166: 827-832.

23 Cullinan P, Harris J, Mills P, et al. Early prescriptions of antibiotics and the risk of allergic disease in adults: a cohort study. Thorax 2004; 59: 11-15.

24 Foliaki S, Nielsen SK, Bjorksten B, et al. Antibiotic sales and the prevalence of symptoms of asthma, rhinitis, and eczema: The International Study of Asthma and Allergies in Childhood (ISAAC). Int J Epidemiol 2004; 33: 558-563.

25 Alm JS, Swartz J, Lilja G, et al. Atopy in children of families with an anthroposophic lifestyle. Lancet 1999; 353: 1485-1488.

26 Cohet C, Cheng S, MacDonald C, et al. Infections, medication use, and the prevalence of symptoms of asthma, rhinitis, and eczema in childhood. J Epidemiol Community Health 2004; 58: 852-857.

27 Droste JH, Wieringa $\mathrm{MH}$, Weyler JJ, et al. Does the use of antibiotics in early childhood increase the risk of asthma and allergic disease? Clin Exp Allergy 2000; 30: 1547-1553.

28 Floistrup H, Swartz J, Bergstrom A, et al. Allergic disease and sensitization in Steiner school children. J Allergy Clin Immunol 2006; 117: 59-66.

29 von Mutius E, Illi S, Hirsch T, et al. Frequency of infections and risk of asthma, atopy and airway hyperresponsiveness in children. Eur Respir J 1999; 14: 4-11.

30 Wickens K, Pearce N, Crane J, et al. Antibiotic use in early childhood and the development of asthma. Clin Exp Allergy 1999; 29: 766-771.

31 Illi S, von Mutius E, Lau S, et al. Early childhood infectious diseases and the development of asthma up to school age: a birth cohort study. BMJ 2001; 322: 390-395.

32 Dom S, Droste JHJ, Sariachvilli MA, et al. Pre- and post-natal exposure to antibiotics and the development of eczema, recurrent wheezing and atopic sensitization in children up to the age of 4 years. Clin Exp Allergy 2010; 40: 1378-1387.

33 Schmitt J, Schmitt NM, Kirch W, et al. Early exposure to antibiotics and infections and the incidence of atopic eczema: a populationbased cohort study. Pediatr Allergy Immunol 2010; 21: 292-300.

34 Celedon JC, Fuhlbrigge A, Rifas-Shiman S, et al. Antibiotic use in the first year of life and asthma in early childhood. Clin Exp Allergy 2004; 34: 1011-1016.

35 Celedon JC, Litonjua AA, Ryan L, et al. Lack of association between antibiotic use in the first year of life and asthma, allergic rhinitis, or eczema at age 5 years. Am J Respir Crit Care Med 2002; 166: 72-75

36 Ponsonby AL, Couper D, Dwyer $\mathrm{T}$, et al. Relationship between early life respiratory illness, family size over time, and the 
development of asthma and hay fever: a seven year follow up study. Thorax 1999; 54: 664-669.

37 Sobko T, Schiott J, Ehlin A, et al. Neonatal sepsis, antibiotic therapy and later risk of asthma and allergy. Paediatr Perinatal Epidemiol 2010; 24: 88-92.

38 Simon MR, Havstad SL, Wegienka GR, et al. Risk factors associated with transient wheezing in young children. Allergy Asthma Proc 2008; 29: 161-165.

39 Thomas M, Custovic A, Woodcock A, et al. Atopic wheezing and early life antibiotic exposure: a nested case-control study. Pediatr Allergy Immunol 2006; 17: 184-188.

40 Verhulst SL, Vael C, Beunckens C, et al. A longitudinal analysis on the association between antibiotic use, intestinal microflora, and wheezing during the first year of life. J Asthma 2008; 45: 828-832.

41 Kummeling I, Stelma FF, Dagnelie PC, et al. Early life exposure to antibiotics and the subsequent development of eczema, wheeze, and allergic sensitization in the first 2 years of life: the KOALA Birth Cohort Study. Pediatrics 2007; 119: e225-e231.

42 Harris JM, Mills P, White C, et al. Recorded infections and antibiotics in early life: associations with allergy in UK children and their parents. Thorax 2007; 62: 631-637.

$43 \mathrm{Su}$ Y, Rothers J, Stern DA, et al. Relation of early antibiotic use to childhood asthma: confounding by indication? Clin Exp Allergy 2010; 40: 1222-1229.

44 Marra F, Marra CA, Richardson K, et al. Antibiotic use in children is associated with increased risk of asthma. Pediatrics 2009; 123: 1003-1010.

45 Martel MJ, Rey E, Malo JL, et al. Determinants of the incidence of childhood asthma: a two-stage case-control study. Am J Epidemiol 2009; 169: 195-205.

46 Kusel MM, de Klerk N, Holt PG, et al. Antibiotic use in the first year of life and risk of atopic disease in early childhood. Clin Exp Allergy 2008; 38: 1921-1928.

47 Kozyrskyj AL, Dahl ME, Ungar WJ, et al. Antibiotic treatment of wheezing in children with asthma: what is the practice? Pediatrics 2006; 117: e1104-e1110.
48 McKeever TM, Lewis SA, Smith C, et al. Early exposure to infections and antibiotics and the incidence of allergic disease: a birth cohort study with the West Midlands General Practice Research Database. J Allergy Clin Immunol 2002; 109: 43-50.

49 Alm B, Erdes L, Mollborg P, et al. Neonatal antibiotic treatment is a risk factor for early wheezing. Pediatrics 2008; 121: 697-702.

50 Mai X-M, Kull I, Wickman M, et al. Antibiotic use in early life and development of allergic diseases: respiratory infection as the explanation. Clin Exp Allergy 2010; 40: 1230-1237.

51 Sterne JA, Egger M, Smith GD. Systematic reviews in health care: investigating and dealing with publication and other biases in meta-analysis. BMJ 2001; 323: 101-105.

52 Sullivan A, Edlund C, Nord CE. Effect of antimicrobial agents on the ecological balance of human microflora. Lancet Infect Dis 2001; 1: 101-114.

53 Noverr MC, Huffnagle GB. The "microflora hypothesis" of allergic diseases. Clin Exp Allergy 2005; 35: 1511-1520.

54 Sudo N, Sawamura S, Tanaka K, et al. The requirement of intestinal bacterial flora for the development of an $\operatorname{IgE}$ production system fully susceptible to oral tolerance induction. I Immunol 1997; 159: 1739-1745.

55 Noverr MC, Huffnagle GB. Does the microbiota regulate immune responses outside the gut? Trends Microbiol 2004; 12: 562-568.

56 Penders J, Stobberingh EE, van den Brandt PA, et al. The role of the intestinal microbiota in the development of atopic disorders. Allergy 2007; 62: 1223-1236.

57 Gronlund MM, Lehtonen OP, Eerola E, et al. Fecal microflora in healthy infants born by different methods of delivery: permanent changes in intestinal flora after Cesarean delivery. I Pediatr Gastroenterol Nutr 1999; 28: 19-25.

58 Kenyon S, Pike K, Jones DR, et al. Childhood outcomes after prescription of antibiotics to pregnant women with preterm rupture of the membranes: 7-year follow-up of the ORACLE I trial. Lancet 2008; 372: 1310-1318.

59 Johnston BC, Supina AL, Ospina M, et al. Probiotics for the prevention of pediatric antibiotic-associated diarrhea. Cochrane Database Syst Rev 2007; 18: CD004827. 